\title{
Power Control in Passive Waveguide Circuits
}

\author{
Jan Zehentner, Jan Machac, Pavel Zabloudil \\ Czech Technical University, Prague, Technicka 2, 16627 Czech Republic \\ Tel: +420-224352273, Fax: +420-224355865, E-mail: zehent@feld.cvut.cz
}

\begin{abstract}
A new concept of a passive waveguide reflection attenuator and a $\mathrm{T}$-junction with a variable ratio of the output powers is reported here. A conductive uniform linear polarization grid of nano thickness is inserted into the standard rectangular waveguide. Rotation of the grid results in a change of the output powers. The measured scattering matrix elements compare well with the computer simulation, confirming the expected characteristics of the two circuits.
\end{abstract}

Index Terms - Power dividers, power combiners, power control, variable attenuators, waveguide attenuators, waveguide T-junctions.

\section{INTRODUCTION}

Setting the power to the required level or dividing the power in microwave systems and measuring setups is a very frequent operation realized by passive or active attenuators and by various power dividers or directional couplers. The operation of these circuits is based on various physical principles and technologies that are used for producing them. Attenuators are fixed or variable. Attenuation is achieved by mechanical movement of the functional part of the attenuator, or it can be controlled electrically. Mechanically controlled attenuators can be of absorbing and reflective types. Greater attenuation is attainable with reflective attenuators. A typical reflective attenuator consists of coaxial input and output sections with a cylindrical waveguide in between. Variations in the length of this central cylindrical section determine the attenuation. However, the fixed installation of the transmission channels does not allow changes in the attenuator length. In the power dividers/combiners there is a wide assortment of circuits. They exhibit either fixed or variable power division. Bethe hole couplers, Schwinger reversed phase couplers, multielement couplers, coupled line directional couplers, branch line and Lange directional couplers, $\mathrm{T}$ junctions, Wilkinson dividers, magic $\mathrm{T}$ and hybrid rings are well-known power dividers [1]. Passive power dividers suffer because the output powers are not easy to change.

Let us consider only circuits in waveguide technology. When a fine linear conductive grid of nano-thickness is inserted into a rectangular waveguide, an interesting response of the propagating dominant mode occurs. This paper reports a new design of a reflective attenuator and a $\mathrm{T}$-junction. The substance of the design consists in the combination of the polarization grid and the waveguide housing. The two circuits were designed and manufactured, and their function was verified by measurements. The administration of the patent and the utility model confirms the contribution of the new power control concept.

\section{CONCEPT OF POWER CONTROL}

Let the linearly polarized electromagnetic plane wave be incident perpendicularly to the set of conductive wires arranged in parallel. The wires represent a linear polarization grid, which at the moment is assumed to be lossless. When the width of the wires of negligible thickness, and the distance from each other, is properly designed, the magnitude

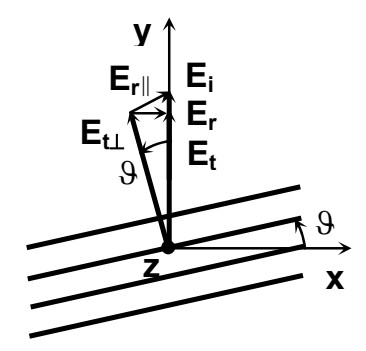

Fig. 1 Perpendicular incidence of a wave on the grid.

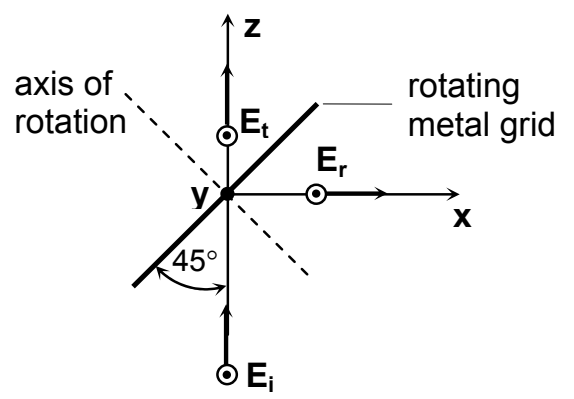

Fig. 2 Oblique incidence of a wave on the grid. 
of the transmitted wave depends on the angle between the plane of the wave polarization and the orientation of the wires. Clearly, according to Fig. 1 the transmitted wave $\mathbf{E}_{\mathrm{t} \perp}=\mathbf{E}_{\mathrm{i}} \cos \vartheta$ and the reflected wave $\mathbf{E}_{\mathrm{r} \|}=\mathbf{E}_{\mathrm{i}} \sin \vartheta$. When we insert the grid into a rectangular waveguide, allowing the dominant mode to propagate, the housing acts as a polarizer admitting propagation only of this mode. Consequently, the wave passing through the grid is $\mathbf{E}_{\mathbf{t}}=\mathbf{E}_{\mathrm{i}} \cos ^{2} \vartheta$, while the wave reflected from the grid is $\mathbf{E}_{\mathrm{r}}=\mathbf{E}_{\mathrm{i}} \sin ^{2} \vartheta$. Turning the grid leads to various magnitudes of transmitted and reflected power. The grid also has similar behavior when the wave is incident obliquely to the grid under an angle, particularly under $45^{\circ}$ as shown in Fig. 2. The perpendicular incidence of a wave on a grid in a waveguide forms the basis of the reflective attenuator. The oblique incidence of a wave on the grid in a waveguide enables the construction of a $\mathrm{T}$ junction with a variable ratio of output powers.

\section{T-JUNCTION WITH A VARIABLE RATIO OF OUTPUT POWERS}

Waveguide T-junctions are used for power dividing and combining. They have two forms, known as $\mathrm{T}$-junctions in the $\mathrm{H}$ plane and $\mathrm{T}$ junctions in the E plane. We are interested from now on in the $\mathrm{T}$-junction in the $\mathrm{H}$ plane when the main guide with ports 1 and 2, and a side guide with port 3, are connected in parallel, as shown in Fig. 3. The junction has two planes of symmetry, $F_{1}$ and $F_{2}$. An inductive diaphragm located in the main guide lying in plane $\mathrm{F}_{2}$ improves the impedance matching of the junction, widens its frequency band and ensures equal output power at port 1 and 2 when port 3 is fed.

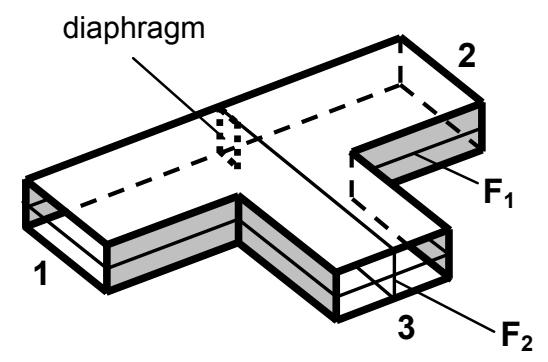

Fig. 3 Compensated T-junction in the H plane.

However this modification of the junction, known as a compensated $\mathrm{T}$-junction, allows no additional change of its parameters, particularly no change of the output power ratio. A new concept of the junction removes this drawback. The metal polariza-

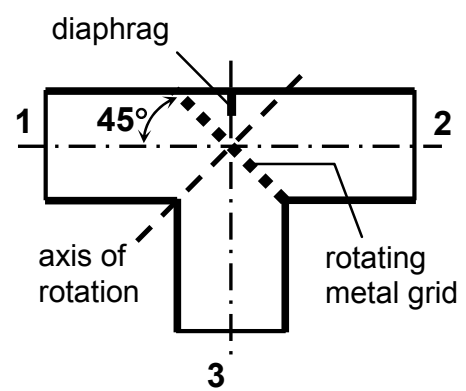

Fig. 4 Arrangement of the rotating grid in the T-junction

tion grid is inserted under an angle of $45^{\circ}$ in the main guide. The grid rotates round its axis perpendicular to the grid-plane, as shown in Fig. 4. Assuming a lossless structure, the power incident on the grid passes, if the conductors of the grid are perpendicular to the $\mathbf{E}$ field direction of the dominant mode in the rectangular waveguide. The power is reflected when the grid conductors are parallel to E. For other orientations of the grid conductors, i. e., apart from the two extreme cases mentioned above, part of the power passes through the grid while the rest of the power is reflected under the incident angle, i. e., $45^{\circ}$.

We designed the junction by means of the CST Microwave Studio (MWS) electromagnetic solver. The investigated structure was modeled in each successive step and the elements of the scattering matrix were observed in the frequency band from 8 to $12 \mathrm{GHz}$. The size of the diaphram resulted from a comparison of the scattering coefficients of the empty junction and the compensated junction. It turned out that the insertion depth of the diaphram is not critical, neither is its thickness when it is less than $1 \mathrm{~mm}$. Consequently we selected the length of the diaphram $6 \mathrm{~mm}$ with a thickness of $0.3 \mathrm{~mm}$. The grid must be located in a cavity between two flanges inclined by $45^{\circ}$ to the main and side guide axes. In the interface plane of two flanges a short at the circumference of the waveguide walls is needed. A high frequency grooved choke was therefore added. A comparison of Fig. 5 and 6 illustrates the influence of the presence of the choke.

In the junction we used a uniform polarization grid, since it turned out from the simulation that uniform and non-uniform allocation of the grid strips/slots provided practically the same scattering matrices. The final selected width of the strips was $100 \mu \mathrm{m}, 60 \mathrm{~nm}$ in thickness, with $100 \mu \mathrm{m}$ wide slots in between. The results of the T-junction simulation confirmed its expected function, therefore it was manufactured and tested. 


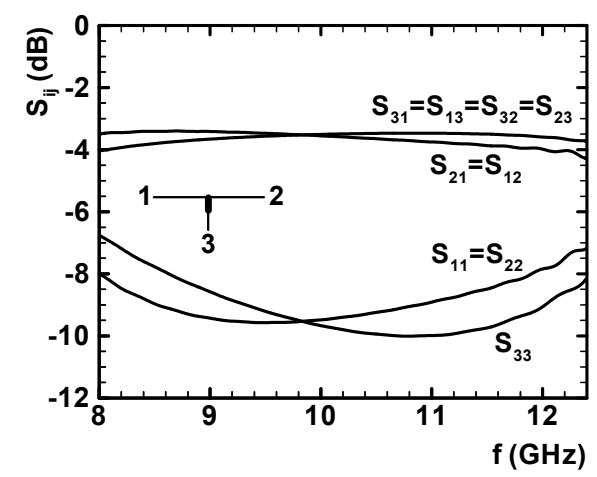

Fig. 5 Scattering coefficients of the compensated Tjunction.

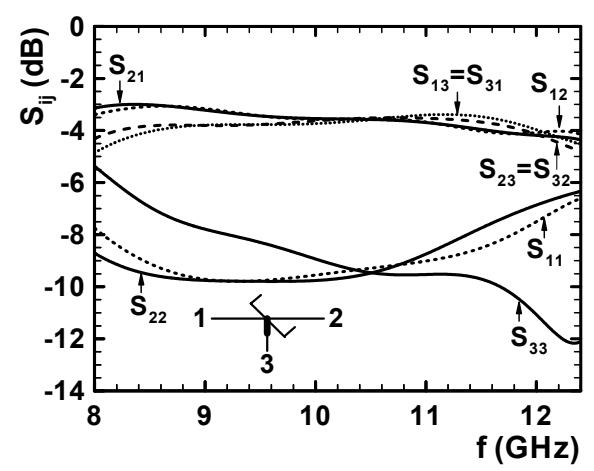

Fig. 6 Scattering coefficients of the compensated Tjunction with a grooved choke located in the plane deviated by $45^{\circ}$ from the main waveguide axis.

\section{REFLECTIVE ATTENUATOR}

The structure of the attenuator is very simple. A conductive uniform polarization grid is inserted into the rectangular waveguide perpendicular to its axis. The grooved choke in the interface plane of two flanges enables rotation of the grid. The same structure of the grid was used as in the case of the Tjunction. Simulation at the MWS confirmed the expected function of the attenuator. The attenuator was then manufactured and measured.

\section{RESUlts OF the MEASUREMENTS}

In the measurements of the reflection and transmission coefficients we used a matched load with $\mathrm{VSWR}<1.08$ from 8.5 to $12.5 \mathrm{GHz}$. Two coaxial/waveguide adaptors connected back-to-back in series had reflection losses less than $-30 \mathrm{~dB}$ and insertion losses less than $-0.2 \mathrm{~dB}$ in the 8.2-12.5 $\mathrm{GHz}$ band. The output powers of a circuit under test are frequency dependent, so from now on we show the relevant quantities only at selected frequencies. The metal polarization grid was made by photo etching a gold layer $60 \mathrm{~nm}$ in thickness sputtered on a polyethyleneterephthalate foil $50 \mu \mathrm{m}$ in thickness.

\section{A. T-junction}

The division of the output powers is frequency dependent in the $8-12 \mathrm{GHz}$ band, as indicated the calculated patterns in Figs. 7 and 8. The reflection losses at port 1 for three frequencies are plotted in Fig. 9. The change in the power ratio $\mathrm{P}_{2} / \mathrm{P}_{3}$ with the angle of the grid at $10 \mathrm{GHz}$ is shown in Fig 10.

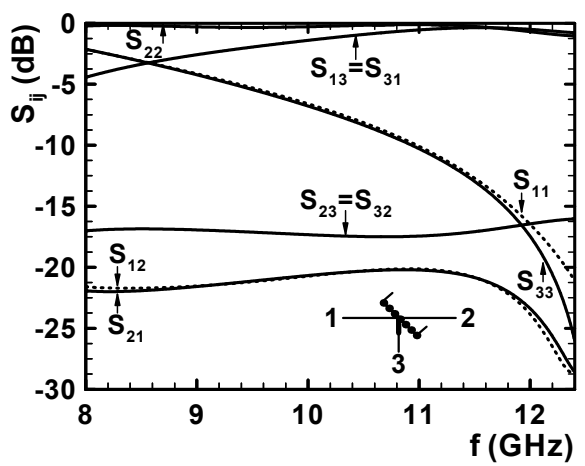

Fig. 7 Scattering coefficients of the T-junction when the grid wires have a vertical orientation.

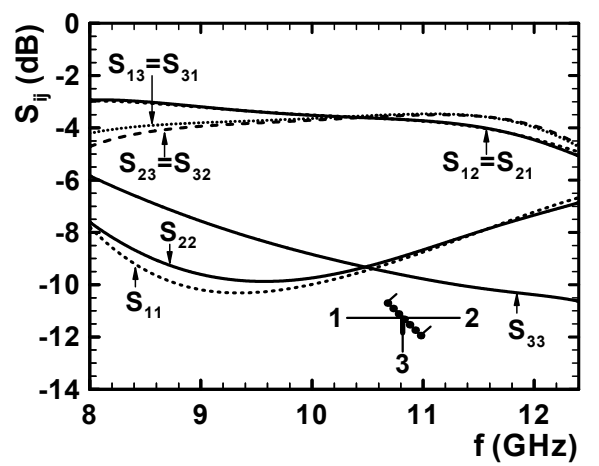

Fig. 8 Scattering coefficients of the T-junction when the grid wires have a horizontal orientation.

The scattering coefficients of the junction confirmed acceptable agreement between the measured values and the simulated frequencydependent values. The functionality of the Tjunction has been verified in practical application [2]- [4].

\section{B. Attenuator}

The attenuator was measured in the same equipment set-up as the T-junction. The measured 


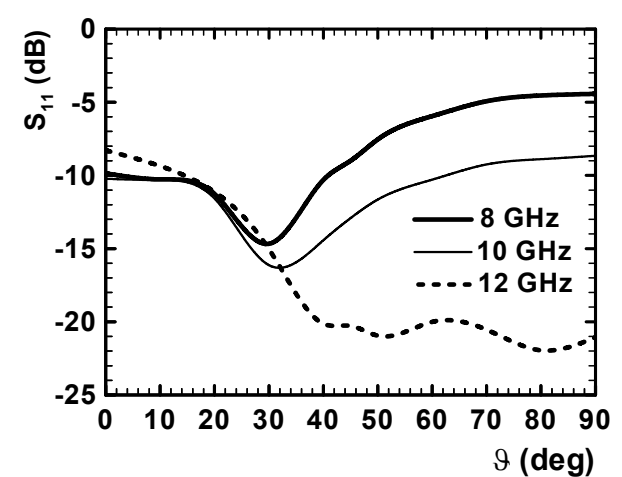

Fig. 9 Reflection losses at fed port 1 for 8,10 and 12 $\mathrm{GHz}$.

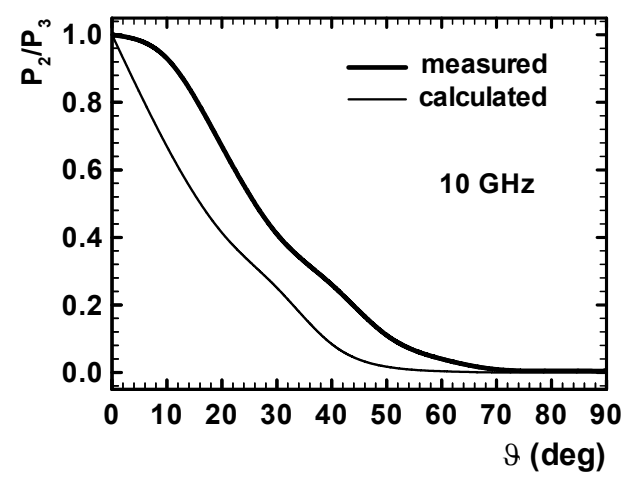

Fig. 10 Calculated and measured ratio of powers $\mathrm{P}_{2} / \mathrm{P}_{3}$ at $10 \mathrm{GHz}$.

transmission coefficient of the attenuator is plotted in Fig. 11, along with the same quantity calculated for an ideally lossless circuit. The highest achievable attenuation decreases with frequency. Consequently, at higher frequencies a denser grid is needed in order to maintain the same attenuation.

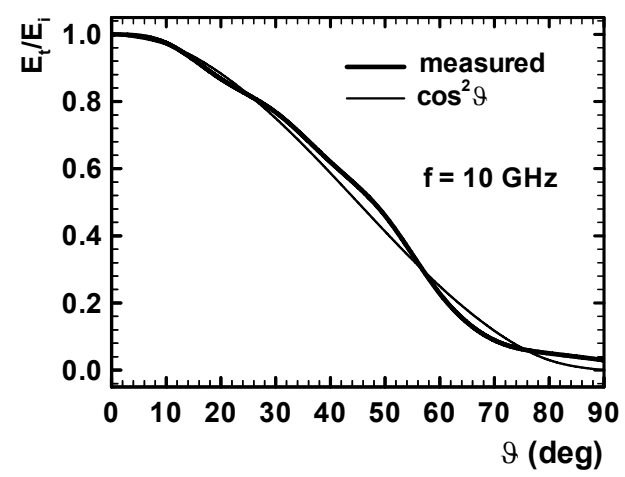

Fig. 11 Measured and calculated transmission coefficient of the attenuator at $10 \mathrm{GHz}$.

\section{CONCLUSION}

The two passive circuits presented here, a Tjunction and a reflective attenuator, provide an opportunity to design a new class of waveguide circuits taking advantage of present-day technologies. Combining waveguides with nano grids improves the parameters of these circuits. Careful, mechanical design and realization ensures their correct, reliable and reproducible operation. Present-day machine tools and standard photolithography are sufficient to produce the housing of the circuits and polarization grids. We believe that they can spread successfully in practical applications.

\section{ACKNOWLEDGEMENT}

This work has been supported by the Grant Agency of the Czech Republic under project 102/06/1106 "Metamaterials, nanostructures and their applications"

\section{REFERENCES}

[1] R. E. Collin, Foundations for Microwave Engineering, Second Edition.,New York: J. Wiley \& Sons, 1992.

[2] J. Zehentner, Waveguide T-junction, Patent No. 296719, Prague: Industrial property office, April 11, 2006.

[3] J. Zehentner, Reflection attenuator with adjustable attenuation, Patent pending No. 2005-790, Prague: Industrial property office, December 19, 2005.

[4] J. Zehentner, Reflection attenuator with adjustable attenuation, Utility model No. 16386, Prague: Industrial property office, March 27, 2006. 